'Facultad de Medicina Clínica Alemana-Universidad Del Desarrollo.

${ }^{2}$ Servicios de Medicina Interna Hospital Padre Hurtado y Clínica Alemana de Santiago, Chile. ${ }^{a}$ Becada Medicina Interna.

Recibido el 15 de julio de 2014, aceptado el 12 de noviembre de 2014 .

Correspondencia a: Dra. Natalia Gallardo Esperanza 2150, San Ramón, Santiago, Chile.

Natalia.gallardoc@gmail.com

\section{Neumomediastino y necrosis cutánea en asociación a dermatomiositis: presentación de un caso clínico y revisión de la literatura}

\author{
NATALIA GALLARDO C. ${ }^{1, a}$, OMAR VALENZUELA L. ${ }^{2}$, \\ SEBASTIÁN IBÁÑEZ V. ${ }^{2}$
}

\section{Pneumomediastinum and cutaneous necrosis in dermatomyositis. Report of one case}

We report a 37 years old male with a dermatomyositis treated with oral cyclophosphamide. He was admitted to the hospital due to a zone of skin necrosis with purulent exudate, located in the second left toe. A complete blood count showed a leukocyte count of 2,600 cells $/ \mathrm{mm}^{3}$. A Chest CAT scan showed a pneumomediastinum with emphysema of adjacent soft tissue. Cyclophosphamide was discontinued and leukocyte count improved. The affected toe was amputated and a chest CAT scan showed a partial resolution of the pneumomediastinum. We discuss and review the pathogenesis, clinical presentation and management of pneumomediastinum and cutaneous necrosis in association with dermatomyositis.

(Rev Med Chile 2015; 143: 120-123)

Key words: Dermatomyositis; Mediastinal emphysema; Skin ulcer; Vascular diseases.
L a dermatomiositis (DM) es una enfermedad del tejido conectivo (ETC) de etiología incierta y compromiso multisistémico, que involucra principalmente el sistema músculoesquelético y la piel. Se asocia a complicaciones respiratorias, siendo la principal la enfermedad pulmonar difusa (EPD), presente en 5 a $30 \%$ de los pacientes con $\mathrm{DM}^{1}$.

Se ha descrito la presencia de neumomediastino $(\mathrm{Nm})$ como una complicación rara en varios tipos de enfermedades del tejido conectivo ${ }^{2}$. El primer caso asociado a DM fue descrito el año $1986^{3}$, y desde entonces se han descrito alrededor de 80 casos, lo cual ha hecho que la DM sea la enfermedad del tejido conectivo más frecuentemente asociada a $\mathrm{Nm}$ espontáneo. Además, se ha encontrado una asociación entre DM, Nm y la presencia de lesiones cutáneas ${ }^{4,5}$.

Describimos el caso de un paciente con DM, necrosis cutánea y $\mathrm{Nm}$. Se realiza una breve revisión de la literatura acerca de la fisiopatología y características clínicas de este grupo de pacientes.

\section{Caso clínico}

Hombre de 37 años, soldador, casado con dos hijos, con DM diagnosticada a los 35 años en otro centro hospitalario, debido a debilidad muscular proximal, creatinquinasa $(\mathrm{CK})$ elevada, electromiografía y biopsia de piel compatible. Sin antecedentes de tabaquismo, consumo de alcohol, drogas, ni otras enfermedades. Recibió corticoides y azatioprina, que suspendió en contexto de artritis séptica de rodilla izquierda. Posteriormente recibió metotrexato, que se suspendió por anemia. Inició ciclofosfamida oral y, por cambio de domicilio, inició controles en el servicio de reumatología de Hospital Padre Hurtado tras dos 
Neumomediastino y necrosis cutánea en dermatomiositis - N. Gallardo et al

años de enfermedad. Al ingreso presentaba atrofia muscular severa, índice de masa corporal de 16, con debilidad muscular de predominio proximal, M2 en extremidades inferiores y M3 en superiores, que impresionó ser determinada más por la atrofia que por actividad de la enfermedad. Además se evidenció una placa necrótica que comprometía la zona distal del segundo dedo del pie izquierdo, con exudado purulento, de larga data, que había requerido numerosas curaciones por enfermería, sin lograr sanar. Sin Raynaud asociado. Se hospitalizó por sospecha de compromiso infeccioso del ortejo. Del laboratorio destacó hematocrito $36,3 \%$, hemoglobina $12,4 \mathrm{mg} / \mathrm{dL}$, leucocitos 2.600 , plaquetas 243.000 , PCR 6 , creatinina 0,36 , BUN 14, CK normal. Las radiografías objetivaron una osteomielitis por lo que se procedió a la amputación del ortejo comprometido. Además se realizó tomografía axial computada (TAC) de tórax, abdomen y pelvis, en el contexto del estudio de su miopatía, que reveló un extenso Nm (Figura 1) asociado a enfisema de partes blandas adyacentes, leve neumoperitoneo, engrosamiento reticular subpleural y bilateral de las bases, determinado probablemente por una EPD incipiente, junto a

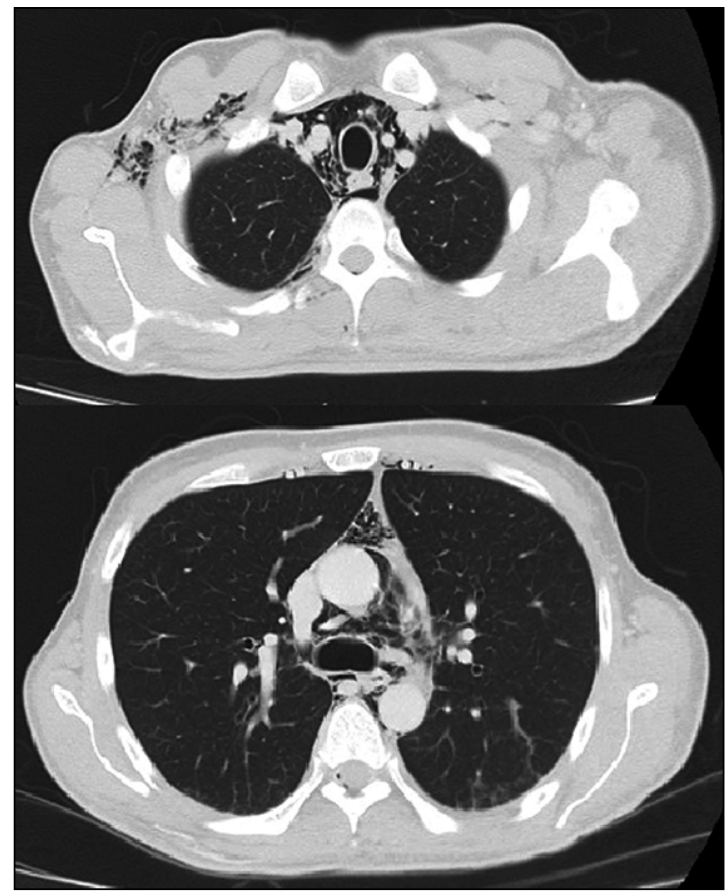

Figura 1. calcificaciones difusas en el tejido celular subcutáneo. Además, se realizó colonoscopia y endoscopia, ambas normales. Tras la suspensión de la ciclofosfamida recuperó la leucopenia. Se realizó biopsia muscular que reveló fibras musculares de tamaño irregular, con acentuados signos de atrofia y células inflamatorias perimisiales. Anticuerpos antinucleares, anti DNA, anti citoplasma de neutrófilo, anti cardiolipinas, anti B2 glicoproteina $1 \mathrm{y}$ anti antígenos nucleares extractables estaban ausentes, al igual que el anticoagulante lúpico. Tras el alta se inició tratamiento con hidroxicloroquina, azatioprina, nifedipino, sildenafil y aspirina, con buena respuesta inicial pero presentó una nueva lesión cutánea, de similares características a la anterior, en el $5^{\circ}$ ortejo del pie derecho (Figura 2). Se intentó tratamiento médico pero evolucionó con compromiso óseo por lo que se realizó amputación de la falange distal. La TAC de tórax de control mostró disminución del $\mathrm{Nm}$. Luego de esto el paciente presentó una buena evolución clínica, IMC 24, sin debilidad muscular, CK normal, ni compromiso respiratorio. Sólo persisten lesiones en piel, superficiales, no necróticas, que han aparecido en otras zonas.

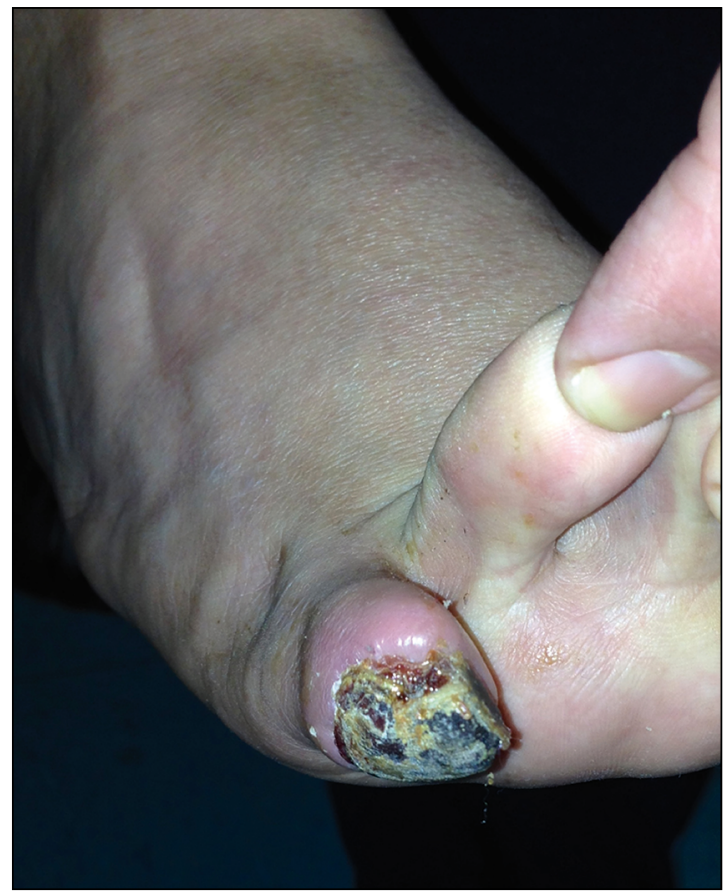

Figura 2. 


\section{Discusión}

Se presenta el caso de un paciente que sufre de DM clásica, con compromiso muscular y de piel objetivado, con lesiones necróticas cutáneas asociadas, en quien, como hallazgo de estudio buscando neoplasia asociada, se evidenció $\mathrm{Nm}$.

Le Goff publicó el año 2009 la mayor revisión de casos de $\mathrm{Nm}$ espontáneo en miopatías inflamatorias, estimando una prevalencia de 2,2\% de $\mathrm{Nm}$. Además encontraron que en pacientes con cualquier ETC y Nm, $80 \%$ presentaba DM, y de estos, 50\% eran DM amiopática ${ }^{2}$.

En cuanto a la fisiopatología se han propuesto tres mecanismos. La primera teoría postula que el $\mathrm{Nm}$ resulta de la ruptura de los alveolos por un aumento de la presión intraalveolar. En el caso de una EPD severa, se pueden formar bulas subpleurales o paracardíacas, las cuales al romperse producen salida de aire hacia el mediastino ${ }^{3}$.

La segunda teoría plantea que el compromiso vascular asociado a la DM puede producir necrosis de la pared bronquial, produciendo $\mathrm{Nm}$ en forma secundaria $(4,5)$. Kono publicó un reporte de 28 pacientes con DM y 20 con polimiositis (PM), en que 4 de los con DM, y ninguno con PM, presentaban $\mathrm{Nm}, \mathrm{y}$, de estos 4, 3 tenían vasculopatía cutánea asociada. Si bien estos 4 casos tenían EPD asociada, en la revisión de la literatura que realizaron de 13 casos de Nm en DM, 5 tenían vasculopatía cutánea, y sólo 2 de estos tenían $\mathrm{EPD}$, por lo que los autores plantearon que, como en los pacientes con DM se observa una abundante infiltración de células B y T CD4 (+) en las regiones perivasculares, la fisiopatología de esta enfermedad estaría ligada a un importante componente vasculopático, no presente en la PM, que explicaría la mayor incidencia de $\mathrm{Nm}$ en los pacientes con $\mathrm{DM}$, y que el $\mathrm{Nm}$ no se relacionaría necesariamente a $\mathrm{EPD}^{4}$. Cabe destacar que en biopsias de piel de 36 pacientes con necrosis o úlceras cutáneas, sólo una demostró vasculitis. El resto reveló microvasculopatía obliterativa con engrosamiento de paredes vasculares, obstrucción o estrechez de lumen, o infiltración linfocítica perivascular6.

Por último, se ha postulado que los corticoides podrían tener un rol en el desarrollo de $\mathrm{Nm}$ al debilitar los tejidos intersticiales, ya que la mayoría de los casos reportados de Nm en DM ocurren en pacientes que están recibiendo terapia esteroidal ${ }^{7}$.
Sin embargo, existen reportes de pacientes que han tenido esta patología sin estar expuestos a corticoides $^{8}$, por lo cual se ha planteado que probablemente esta asociación refleja que quienes realizan Nm espontáneo suelen ser pacientes con enfermedad más severa, y expuestos a terapia más agresiva desde un comienzo ${ }^{2}$.

Los factores de riesgo propuestos que predispondrían a los pacientes con DM a desarrollar Nm son la presencia de EPD, de vasculopatía cutánea, el uso previo de corticoides y presentar CK normal al momento del diagnóstico?.

En cuanto al pronóstico, algunos autores han sugerido que el $\mathrm{Nm}$ espontáneo es un factor de mal pronóstico para pacientes con DM. Sin embargo, los casos reportados en la literatura que han tenido un outcome desfavorable han ocurrido principalmente en pacientes con una EPD asociada ${ }^{10}$. Yoshida sugirió que el $\mathrm{Nm}$ no es un factor de mal pronóstico en sí mismo, sino que es la presencia y la severidad de la EPD la que dicta el pronóstico ${ }^{11}$. Le Goff evaluó la sobrevida de pacientes con DM y Nm, al año y dos años fue de $64 \%$ y $55 \%$ respectivamente, y los factores de mal pronóstico fueron la ausencia de debilidad muscular, la capacidad vital disminuida al ingreso y la disminución en la capacidad de difusión de monóxido de carbono, lo cual es concordante con la apreciación previamente descrita, que es que los pacientes con EPD más severa son los que tienen mayor mortalidad ${ }^{2}$. Incluso, se observa que los pacientes que se presentan sin EPD asociada tienen un curso benigno sin necesidad de una terapia específica, como en nuestro paciente, que tenía hallazgos dudosos de EPD en el TAC de tórax y el neumomediastino se resolvió en forma espontánea sin ninguna intervención en particular.

No hay recomendaciones acerca de una terapia específica en pacientes con Nm y DM. Se ha descrito en la literatura el uso de corticoides y otros agentes inmunosupresores, como ciclosporina, metotrexato, azatioprina y ciclofosfamida, con resultados dispares ${ }^{2,10}$. Hay un reporte de uso de rituximab con buena respuesta en una paciente con DM refractaria a terapia, asociada a $\mathrm{Nm}$ y neumotórax espontáneo ${ }^{12}$. Sin embargo, estas terapias han estado orientadas por las patologías asociadas (en particular EPD), más que por el $\mathrm{Nm}$ en si mismo. Hay casos en los cuales no se ha hecho ninguna intervención y han presentado una buena evolución clínica ${ }^{8}$. Por lo tanto, la terapia 
se deberá definir caso a caso, y en relación a las patologías concomitantes.

En conclusión se presenta un paciente con DM, Nm y necrosis, buena evolución clínica, probablemente por la ausencia de una EPD severa. Es necesario aumentar el índice de sospecha de esta patología en pacientes con DM, y así lograr tener mayor claridad acerca de la fisiopatología, factores pronósticos y terapia óptima.

\section{Referencias}

1. Powell C, Kendall B, Wernick R, Heffner J. A 34-year-old Man With Amyopathic Dermatomyositis and Rapidly Progressive Dyspnea With Facial Swelling. Chest 2007; 132: 1710-3.

2. Le Goff B, Chérin P, Cantagrel A, Gayraud M, Hachulla E, Laborde F, et al. Pneumomediastinum in Interstitial Lung Disease Associated With Dermatomyositis and Polymyositis. Arthritis Rheum 2009; 61: 108-18.

3. Bradley J. Spontaneous Pneumomediastinum in Adult Dermatomyositis. Ann Rheum Dis 1986; 45: 780-2.

4. Kono H, Inokuma S, Nakayama H, Suzuki M. Pneumomediastinum in Dermatomyositis: Association With Cutaneous Vasculopathy. Ann Rheum Dis 2000; 59: 372-6.
5. Cicuttini F, Fraser K. Recurrent Pneumomediastinum in adult dermatomyositis. J Rheumatol 1989; 16: 384-6.

6. Kawachi Y, Maruyama H, Furuta J, Fujisawa Y, Nakamura Y, Takahashi T, et al. Cutaneous deep necrosis with dermatomyositis: correlation with intersticial pneumonia. Eur J Dermatol 2007; 17 (4): 345-6.

7. Barvaux V, Van Mullen X, Piertes T, Houssiau F. Persistent pneumomediastinum and dermatomyositis: A case report and review of the literature. Clin Rheumatol 2001: 20; 359-61.

8. Isfer RS, Nakao SK, Cernea SS. Dermatomyositis associated with pneumomediastinum and subcutaneous cellular tissue emphysema. Br J Rheumatol 1994; 33: 1092.

9. Korkmaz C, Ozkan R, Akay M, Hakan T. Pneumomediastinum and subcutaneous emphysema associated with dermatomyositis. Rheumatology 2001; 40: 476-8.

10. Tang R, Millet C, Green J. Amyopathic Dermatomyositis Complicated by Pneumomediastinum. Clin Aesthetic 2013; 6: 40-3.

11. Yoshida K, Kurosaka D, Kingetsu I, Hirai K, Yamada A. Pneumomediastinum in dermatomyositis itself is not a poor prognostic factor: report of a case and review of the literature. Rheumatol Int 2008; 28: 913-7.

12. Lee M, Hutchinson D. Spontaneous pneumomediastinum secondary to refractory dermatomyositis successfully treated with rituximab. Clin Rheumatol 2010. 